\title{
HIGH-SPEED MICROCHIP ELECTROPHORESIS: EXPLORING THE LIMITS
}

\author{
Stephen C. Jacobson, Christopher T. Culbertson, and J. Michael Ramsey \\ Oak Ridge National Laboratory \\ P.O. Box 2008 \\ Oak Ridge, TN 37831-6142
}

\begin{abstract}
Microchip electrophoresis was used to resolve a binary mixture in $0.8 \mathrm{~ms}$ using a separation field strength of $53 \mathrm{kV}$ $\mathrm{cm}^{-1}$ and a separation length of $200 \mu \mathrm{m}$. The microchip design permitted an electric field strength of $6.1 \mathrm{~V} \mathrm{~cm}^{-1}$ in the separation channel per volt applied to the microchip. Concurrently, the spatial extent of the injection plug and the detector observation region were minimized to increase separation efficiency. Plate counts were generated at rates up to 260,000 plates $s^{-1}$.
\end{abstract}

\section{INTRODUCTION}

Microfabricated substrates are rapidly becoming a convenient platform with which to execute liquid phase analysis, and consequently, a variety of techniques have been implemented on microchips. Capillary electrophoresis [1, $2,3,4$ ], synchronized cyclic electrophoresis [5], free-flow electrophoresis [6], and capillary gel electrophoresis [7,8] have been demonstrated for the separation of ions. Coupling of sample handling and reactions with separation techniques can provide complete chemical analysis, i.e., the Lab-on-aChip concept. Some simple monolithically integrated devices have appeared in the literature and include pre- $[9,10]$ and postcolumn [11,12] chemical reactions in conjunction with electrophoretic separations. In other work, a hybridized device combined a microfabricated silicon thermal cycler for PCR amplification and a glass microchip for product analysis [13]. In addition, a monolithic glass device combined multiplexed PCR amplification and electrophoretic product sizing [14]. As more features are integrated into a planar format, performance optimization becomes essential, especially if pursuing high-speed analysis.

In this paper we describe instrumentation for submillisecond electrophoretic separations representing an $\approx$ 100-fold decrease in analysis time over previous methods. High-speed electrophoretic separations have been performed in capillaries using optical gating for sample introduction [15], and on microchips using a simple cross geometry [3]. Both approaches had analysis times on the order of $100 \mathrm{~ms}$. Several issues must be addressed to enhance the separation performance in capillary electrophoresis. Most importantly, the separation field strength must be optimized to reduce the analysis time and minimize dispersion due to diffusion and thermal convection. In addition, the spatial extent of the injection plug and detector observation region must be minimized. The injection plug length can be minimized by fabricating narrow channel dimensions for the injection valve and confining the sample volume within the injection cross using electric fields. Similarly, the detector observation length can be minimized for fluorescence detection by having a small excitation volume or tight spatial filtering. The channel manifold can be designed to reduce the potential drop in areas not contributing to the separation so that high separation field strengths can be achieved with modest applied potentials. To achieve this design goal in a single etch step, narrow channels were fabricated for the injection valve and separation channel, and wide channels for all other sections of the channel manifold. This enabled separation field strengths of $6.1 \mathrm{~V} \mathrm{~cm}$ ' per volt of applied potential.

\section{EXPERIMENTAL SECTION}

The microchips were fabricated using standard photolithographic, wet chemical etching and bonding techniques as described previously. Briefly, a photomask was fabricated by sputtering chrome $(30 \mathrm{~nm})$ and gold $(100 \mathrm{~nm})$ onto a glass slide, spinning a positive photoresist onto the gold film, and exposing the microchip design (Figure 1) into the photoresist using a CAD/CAM laser machining system (argon ion, $457 \mathrm{~nm}$ ). Subsequently, the metal films were etched using $\mathrm{KI} / \mathrm{I}_{2}$ for $\mathrm{Au}$ and $\mathrm{K}_{3} \mathrm{Fe}(\mathrm{CN})_{6} / \mathrm{NaOH}$ for $\mathrm{Cr}$. The channel design was then transferred onto glass substrates using a positive photoresist and UV exposure. After developing the photoresist, the chrome film was etched, and then channels were etched into the substrate in a dilute, stirred $\mathrm{HF} / \mathrm{NH}_{4} \mathrm{~F}$ bath. 3.5-mm access holes to the channels were drilled in the substrate followed by bonding of the cover plate to the substrate over the etched channels to form a closed network of channels. Cylindrical glass reservoirs were affixed on the substrate using epoxy.

Control of the high voltage at the four fluid reservoirs was performed using a single high voltage power supply (10A12-P4, Ultravolt) with voltage division in $5 \%$ increments. High voltage relays (K81C245, Kilovac) were used to toggle between the sample loading and separation modes. Input to the power supply and high voltage relays was computer controlled. An average delay of $500 \mu \mathrm{s}$ was observed when switching from the sample loading mode to the separation mode. Platinum electrodes provided electrical contact from the high voltage leads to the solutions in the reservoirs. With this configuration, injection volume was controlled by applying potentials at each of the reservoirs during the injection and separation modes. The sample flowing through the injection valve from the sample to 
sample-waste reservoir was spatially confined by electric fields in the buffer and separation channels. To execute the separation, the voltages are reconfigured with the primary flow from the buffer reservoir to the waste reservoir. The sample and sample-waste reservoirs are maintained at a fraction $(50 \%)$ of the potential applied to the buffer reservoir in the separation mode to prevent leakage of sample into the separation channel from the sample and sample-waste channels. This scheme introduces small spatial extent sample plugs and provides high separation efficiency and volumetric reproducibility ( $<0.3 \%$ relative standard deviation).

Microchip separations were monitored using onmicrochip laser induced fluorescence detection. ${ }^{10}$ An argon ion laser (514.5 nm, $\approx 10 \mathrm{~mW}$; Coherent) was used for excitation and focused to an $\approx 25 \mu \mathrm{m}$ spot onto the separation channel using a lens (200 mm focal length). The fluorescence signal was collected using a $100 x$ (0.7 NA) objective lens (Mitutoyo), followed by spatial filtering $(0.4 \mathrm{~mm}$ diameter pinhole) and spectral filtering (550 nm cut-on), and measured using a photomultiplier tube (PMT; Oriel 77340). The PMT response was amplified (Kiethley $428 \mathrm{M}$ ) and recorded with an oscilloscope (Tektronix TDS350). The data on the oscilloscope was then transferred to a Macintosh computer using a GPIB interface and Labview 4.1 (National Instruments). The compounds used for the experiments were rhodamine $\mathrm{B}$ (RB; $8 \mu \mathrm{M}$; Exciton) and dichlorofluorescein (DCF; $4 \mu \mathrm{M}$ ). The buffer in all of the reservoirs was $1 \mathrm{mM}$ sodium tetraborate and $5 \mathrm{mM} \mathrm{N}$-[2-Hydroxyethyl]piperazineN'-[2-ethanesulfonic acid] (HEPES).

\section{RESULTS AND DISCUSSION}

Estimates of the minimum efficiency, separation length, and separation field strength were needed to design a microchip for high-speed electrophoresis. The minimum number of plates required to resolve rhodamine $B(R B)$ and dichlorofluorescein (DCF) can be calculated from equation 1 [16],

$$
N=\left(\frac{4 R \mu_{a g}}{\mu_{R B}-\mu_{D C F}}\right)^{2}
$$

where $N$ is the number of theoretical plates, $R$ is the resolution, and $\mu_{x}$ are the electrokinetic mobilities for RB, DCF, and their average (avg). For a desired resolution of 1.5 , and the electrokinetic mobilities for RB and DCF of $6.2 \times 10^{-4}$ and $4.0 \times 10^{-4} \mathrm{~cm}^{2} \mathrm{~V}^{-1} \mathrm{~s}^{-1}$, respectively, 185 plates are required.

The separation length, $L_{\text {sep }}$, can then be calculated from equation 2 ,

$$
L_{\text {sep }}=N H_{\text {total }}
$$

where $H_{\text {toral }}$ is the total plate height with contributions from diffusion, injection plug length, $l_{i n}$, and detector observation length, $l_{d e t}$, respectively. $H_{\text {tout }}$ can be calculated from equation 3 ,

$$
H_{\text {toral }}=\frac{2 D t_{D C E}}{L_{\text {sep }}}+\frac{l_{i n j}^{2}}{16 L_{\text {sep }}}+\frac{l_{\mathrm{dct}}^{2}}{12 L_{\text {sep }}}
$$

where $D$ is the diffusion coefficient $\left(3 \times 10^{-6} \mathrm{~cm}^{2} \mathrm{~s}^{-1}\right)$, and $t_{D C F}$ is the migration time of the slower component $(1 \mathrm{~ms})$. The sample plug injected onto the separation channel is assumed to be a Gaussian function, and for this calculation a baseline width $(4 \sigma)$ of $30 \mu \mathrm{m}$ was used. The detector observation length was considered to be a rectangular function defined by the spatial filter and is $4 \mu \mathrm{m}$. Using these assumed values in equation 3 results in an estimated separation length of $\approx 100$ $\mu \mathrm{m}$. Lastly, the separation field strength, $E_{\text {sep }}$, required to resolve these components in under $1 \mathrm{~ms}$ can then be estimated by equation 4 ,

$$
E_{\text {sep }}=\frac{L_{\text {sep }}}{t_{D C F} \mu_{D C F}}
$$

yielding a value of $26 \mathrm{kV} \mathrm{cm}$.

Figure 1 shows a schematic of the microchip designed to meet these criteria. Wide channels were used to minimize the potential drop from the reservoirs to the injection valve and separation channel. Figure 2 shows a photograph of a microchip. The fabrication included a single etch step with constant channel depths of $7.0 \mu \mathrm{m}$. Consequently, the different resistivities per unit length result from different channel widths. The wide channels are $440 \mu \mathrm{m}$ wide, and the narrow channels are $26 \mu \mathrm{m}$ wide. The $26 \mu \mathrm{m}$ linewidth was the narrowest, high quality line that could be produced with our inhouse direct-write system. The relative resistivity between the narrow and wide channels is 16.9 per unit length, and consequently, the potential drop along the wide channel is

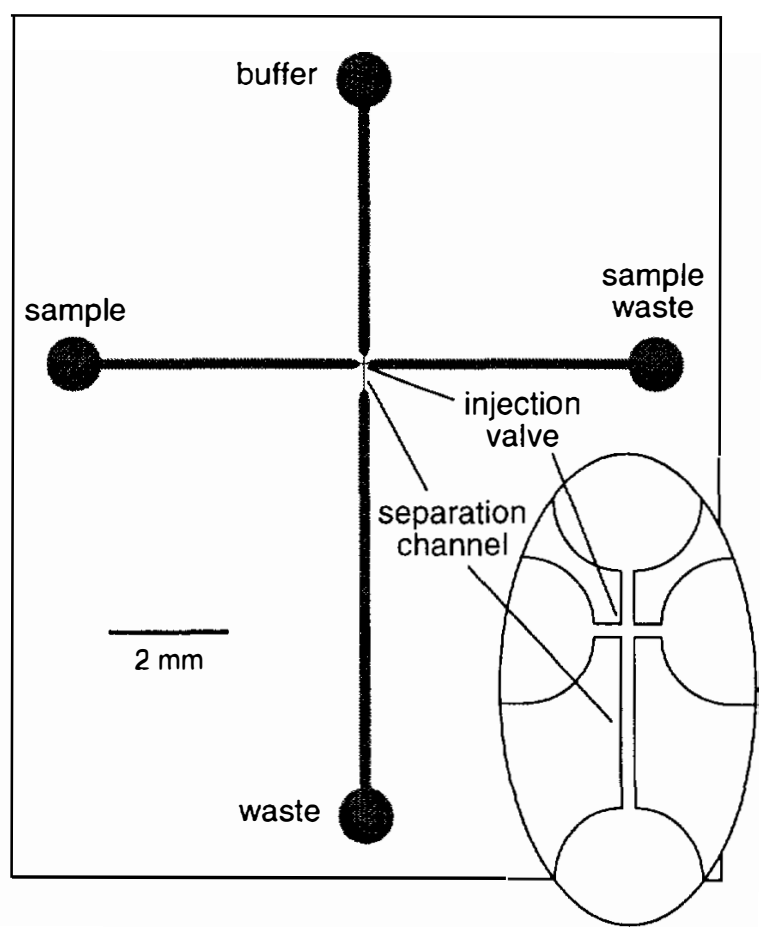

Figure 1. Schematic of microchip used for high-speed electrophoretic separations. Inset. Enlargement of the injection valve and separation channel. 


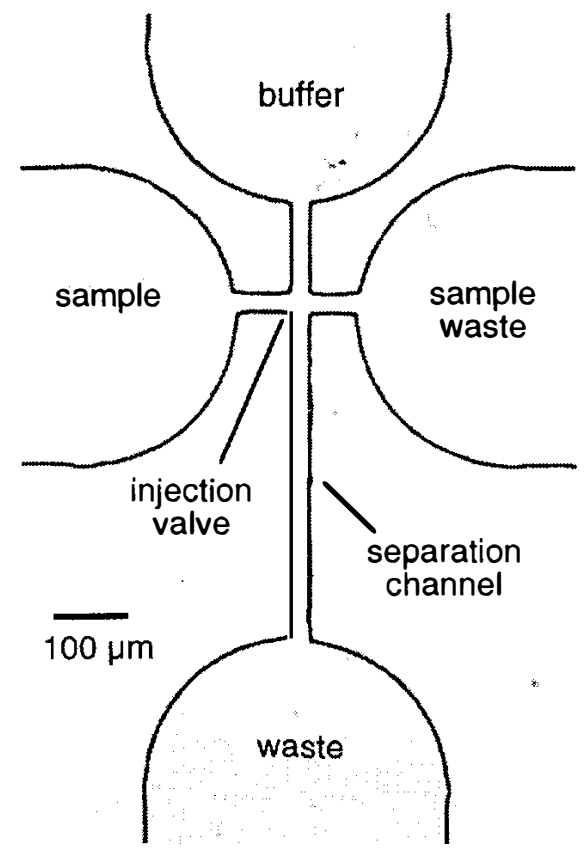

Figure 2. Photograph of microchip used for high-speed electrophoretic separations. The area of the photograph is $0.8 \times 1.2 \mathrm{~mm}$, and the injection valve and separation channel are shown. The wide channels are $440 \mu \mathrm{m}$ wide, and the narrow channels are $26 \mu \mathrm{m}$ wide.

16.9 times less than along the narrow channels for a given length. This design gives a separation field strength of $6.1 \mathrm{~V}$ $\mathrm{cm}^{-1}$ per volt of applied potential. If a microchip of similar dimension were fabricated with constant width channels, the applied potential would have to be over 8 times greater to

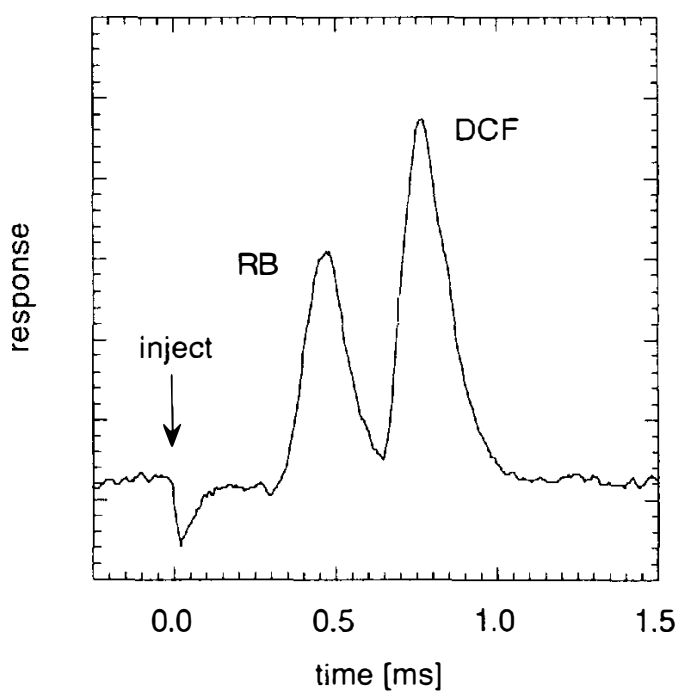

Figure 3. High-speed electropherogram of rhodamine $B(R B)$ and dichlorofluorescein (DCF) resolved in $0.8 \mathrm{~ms}$ using a separation field strength of $53 \mathrm{kV} \mathrm{cm}$ and a separation length of $200 \mu \mathrm{m}$. The start time is marked with an arrow at $0 \mathrm{~ms}$. achieve equivalent separation field strengths. For example, the highest separation field strength used in this work was 53 $\mathrm{kV} \mathrm{cm}{ }^{-1}$ which would require $71 \mathrm{kV}$ of applied potential for a microchip with a uniform width channel design. Such high potentials are clearly not practical.

Figure 3 shows an electrophoretic separation from a single injection of the binary mixture of rhodamine $\mathbf{B}$ and dichlorofluorescein resolved in $0.8 \mathrm{~ms}$ using the microchip depicted in Figure 2. The separation was monitored $200 \mu \mathrm{m}$ downstream from the injection valve, and a separation field strength of $53 \mathrm{kV} \mathrm{cm}^{-1}$ was used (8.6 kV applied to the microchip). The variation of sample velocity with the separation electric field strength is plotted in Figure 4 . The solid lines are calculated from an average of the velocities at the two lowest field strengths (13 and $27 \mathrm{kV} \mathrm{cm}^{-1}$ ) and extrapolated to higher separation field strengths to demonstrate the deviation from linear behavior at higher field strengths (40 and $53 \mathrm{kV} \mathrm{cm}^{-1}$ ). This nonlinear behavior can be attributed to Joule heating of the buffer which lowers the buffer viscosity resulting in higher linear velocities. To lessen the Joule heating in the channels, $83 \%$ of the buffer composition was a zwitterionic organic buffer, HEPES. The heat dissipated in the separation channel for the $53 \mathrm{kV} \mathrm{cm}{ }^{-1}$ separation field strength was estimated to be $172 \mathrm{~W} \mathrm{~m}^{1}$ assuming a potential drop of $2.8 \mathrm{kV}$, a current of $32 \mu \mathrm{A}$, and a length of $520 \mu \mathrm{m}$. This value is considered to be exceedingly high for conventional capillary electrokinetic separations, but the separation efficiency was not adversely affected as discussed below. For the lower separation field strengths (13 and $27 \mathrm{kV} \mathrm{cm}^{-1}$ ), the electroosmotic mobility of $6.21 \times 10^{-4}$ $\mathrm{cm}^{2} \mathrm{~V}^{-1} \mathrm{~s}^{-1}$ was estimated and coincided well with previously reported values [17] which indicated that the calculation of the electric field strength in the separation channel was

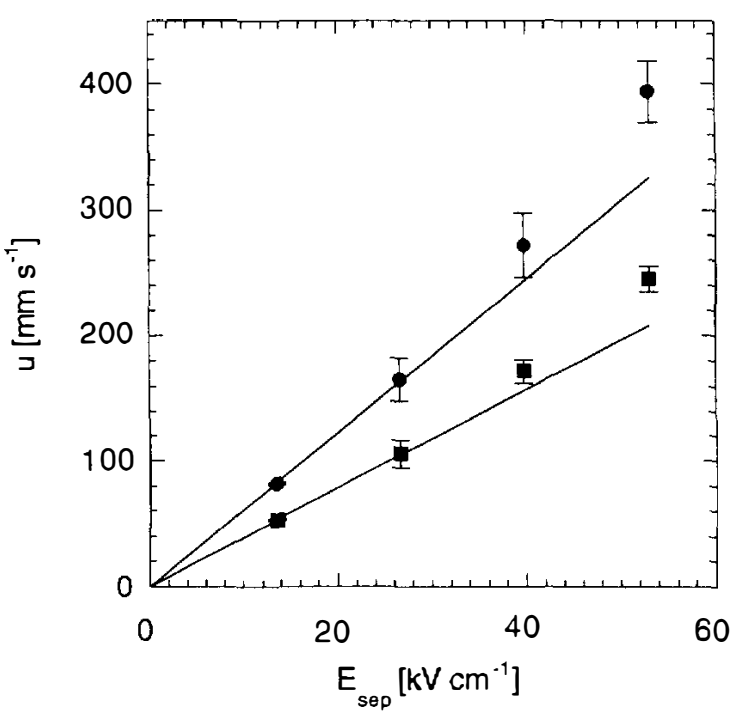

Eigure 4 Variation of linear velocity $(u)$ with separation field strength $\left(E_{\text {sep }}\right)$ for rhodamine $B$ (O) and dichlorofluorescein ( $\boldsymbol{D})$. Lines are calculated from the average mobilities measured at separation field strengths of 13 and $27 \mathrm{kV} \mathrm{cm}^{-1}$. The errors bars are $\pm \sigma$. 
acceptable. Also, the ratio between the electrokinetic mobilities for rhodamine $\mathrm{B}$ and dichlorofluorescein remain nearly constant at 1.58 over the range of separation field strengths. Although the volumetric flow rate is normally considered to be minuscule in electrokinetically driven microchannel devices, the high linear velocities produced here result in a volumetric flow rate of over $4 \mu \mathrm{L} \mathrm{min}{ }^{-1}$, which is two orders of magnitude higher than most microchip assays.

In conclusion, the initial demonstration of submillisecond separations was extremely promising. Straightforward improvements in the separation efficiency and analysis time can be made by reducing the contributions of the injection plug width and Joule heating to the plate height. High-speed microchip electrophoresis could be a useful tool for ultra-high throughput drug discovery, monitoring millisecond time-scale kinetics for chemical and biochemical reactions, or as the final dimension to multidimensional separation systems.

\section{ACKNOWLEDGEMENTS}

This research is sponsored by the U.S. Department of Energy, Office of Research and Development. Oak Ridge National Laboratory (ORNL) is managed by Lockheed Martin Energy Research Corporation for the U.S. Department of Energy under contract DE-AC05-96OR22464. C.T.C. was supported through an appointment to the ORNL Postdoctoral Research Associates Program administered by ORISE and ORNL.

\section{REFERENCES}

1. D.J. Harrison, A. Manz, Z. Fan, H. Lüdi, H.M. Widmer, "Capillary Electrophoresis and Sample Injection Systems Integrated on a Planar Glass Chip," Anal. Chem., 64, 1926 (1992).

2. D.J. Harrison, K. Fluri, K. Seiler, Z. Fan, C.S. Effenhauser, A. Manz, "Micromachining a Miniaturized Capillary Electrophoresis-Based Chemical Analysis System on a Chip," Science, 26I, 895 (1993).

3. S.C. Jacobson, R. Hergenröder, L.B. Koutny, J.M. Ramsey, "High-Speed Separations on a Microchip," Anal. Chem., 66, 1114 (1994).

4. L.B. Koutny, D. Schmalzing, T.M. Taylor, M. Fuchs, "Microchip Electrophoretic Immunoassay for Serum Cortisol," Anal. Chem., 68, 18 (1996).

5. N. Burggraf, A. Manz, C.S. Effenhauser, E. Verpoorte, N.F. de Rooij, H.M. Widmer, "Synchronized Cyclic Capillary Electrophoresis - A Novel Approach to Ion Separations in Solution," J. High Res. Chromatogr., I6, 594 (1993).

6. D.E. Raymond, A. Manz, H.M. Widmer, "Continuous Sample Pretreatment Using a Free-Flow Electrophoresis Device Integrated onto a Silicon Chip," Anal. Chem., 66, 2858 (1994).

7. C.S. Effenhauser, A. Paulus, A. Manz, H.M. Widmer, "High-Speed Separation of Antisense Oligonucleotides on a Micromachined Capillary Electrophoresis Device," Anal. Chem., 66, 2949 (1994).
8. A.T. Woolley, R.A. Mathies, "Ultra-High-Speed DNA Sequencing Using Capillary Electrophoresis Chips," Anal. Chem., 67, 3676 (1995).

9. S.C. Jacobson, R. Hergenröder, A.W. Moore, J.M. Ramsey, "Precolumn Reactions with Electrophoretic Analysis Integrated on a Microchip," Anal. Chem., 66, 4127 (1994).

10. S.C. Jacobson, J.M. Ramsey, "Integrated Microdevice for DNA Restriction Fragment Analysis," Anal. Chem., 68, 720 (1996).

11. S.C. Jacobson, L.B. Koutny, R. Hergenröder, A.W. Moore, J.M. Ramsey, "Microchip Capillary Electrophoresis with an Integrated Postcolumn Reactor," Anal. Chem., 66, 3472 (1994).

12. K. Fluri, G. Fitzpatrick, N. Chiem, D.J. Harrison, Anal. Chem., "Integrated Capillary Electrophoresis Devices with an Efficient Postcolumn Reactor in Planar Quartz and Glass Chips," 68, 4285 (1996).

13. A.T. Woolley, D. Hadley, P. Landre, A.J. deMello, R.A. Mathies, M.A. Northrup, "Functional Integration of PCR Amplification and Capillary Electrophoresis in a Microfabricated DNA Analysis Device," Anal. Chem., 68, 4081 (1996).

14. L.C. Waters, S.C. Jacobson, N. Kroutchinina, J . Khandurina, R.S. Foote, J.M. Ramsey, "Microchip Device for Cell Lysis, Multiplex PCR Amplification, and Electrophoretic Sizing," Anal. Chem., 70, 158 (1998).

15. A.W. Moore Jr., J.W. Jorgenson, "Study of Zone Broadening in Optically Gated High-Speed Capillary Electrophoresis,"Anal. Chem., 65, 3550 (1993).

16. J.C. Giddings, Unified Separation Science, John Wiley \& Sons, Inc., New York (1991).

17. For example, P.K. Dasgupta, S. Liu, "Electroosmosis: A Reliable Fluid Propulsion System for Flow Injection Analysis," Anal. Chem., 66, 1792 (1994). 